\title{
Brilliant violet fluorochromes in simultaneous multicolor flow cytometry-fluorescence in situ hybridization measurement of monocyte subsets and telomere length in heart failure
}

\author{
Santiago Roura 1,2,6, Marco A Fernández ${ }^{3,6}$, Elena Elchinova ${ }^{4,5,6}$, Iris Teubel ${ }^{3}$, Gerard Requena ${ }^{3}$, Roser Cabanes ${ }^{4}$, \\ Josep Lupón $n^{4,5}$ and Antoni Bayes-Genis ${ }^{1,4,5}$
}

Conventional analytical methods to determine telomere length $(\mathrm{TL})$ have been replaced by more precise and reproducible procedures, such as fluorescence in situ hybridization coupled with flow cytometry (flow-FISH). However, simultaneous measurement of TL and cell phenotype remains difficult. Relatively expensive and time-consuming cell-sorting purification is needed to counteract the loss, due to stringent FISH conditions, of prehybridization fluorescence by the organic fluorochromes conventionally used in the phenotyping step. Here, we sought to assess whether the newly developed Brilliant Violet (BV) dyes are valuable to specifically and simultaneously assess the distribution and telomere attrition of monocyte subsets circulating in the blood of a cohort of patients with heart failure. We performed flow-FISH on blood samples from 28 patients with heart failure. To differentiate among monocyte subsets, we used BV and conventional fluorochromes conjugated to antibodies against CD86, CD14, CD16, and CD15. We simultaneously assessed the TLs of the monocyte subsets with a telomere-specific peptide nucleic acid probe labeled with fluorescein isothiocyanate. The BV dyes completely tolerated the harsh conditions required for adequate DNA denaturation and simultaneously provided accurate identification of monocyte subpopulations and respective TLs. The presented protocol may be faster and less expensive than those used currently for purposes such as establishing associations among patient categories, disease progression, monocyte heterogeneity, and aging in the context of heart failure.

Laboratory Investigation (2016) 96, 1223-1230; doi:10.1038/labinvest.2016.100; published online 12 September 2016

Determination of telomere length (TL) is clinically valuable, since it reflects both the number of times the cell has divided and telomere attrition; both of these attributes are associated with the genetic instability, pathogenesis, and progression of a variety of human disorders. Current laboratory techniques for TL measurement include Southern blot, PCR, single TL analysis, and fluorescence in situ hybridization coupled with flow cytometry (flow-FISH). However, these methodologies have critical limitations; for example, they require large sample amounts and antibodies that are preferably conjugated to heat-stable fluorochromes, their analytical sensitivity is suboptimal when very short telomeres are assessed, and measurements among different samples vary excessively. ${ }^{1}$
Flow-FISH is a methodology that has been thoroughly validated for clinical diagnosis, and it includes the option to analyze expression of specific surface antigens in either single cells or cell subsets. ${ }^{2,3}$ However, previous studies have revealed that combining cell phenotyping by flow cytometry with TL by FISH is difficult due to the harsh conditions required for hybridization of the telomere probe (ie, intense fixation and incubation at $82^{\circ} \mathrm{C}$ ). Relatively expensive and time-consuming cell subset purification (eg, by cell-sorting) is required to counteract the loss of fluorescence of the conventional, non-heat-stable, organic fluorochromes that are usually used in the immunophenotyping step. As an alternative, a small group of fluorochromes, including

\footnotetext{
${ }^{1}$ ICREC Research Program, Germans Trias i Pujol Health Science Research Institute, Badalona, Spain; ${ }^{2}$ Center of Regenerative Medicine in Barcelona, Barcelona, Spain; ${ }^{3}$ Flow Cytometry Facility, Germans Trias i Pujol Health Science Research Institute, Badalona, Spain; ${ }^{4}$ Cardiology Service, Germans Trias i Pujol University Hospital, Badalona, Spain and ${ }^{5}$ Department of Medicine, Universitat Autònoma de Barcelona, Barcelona, Spain

Correspondence: Dr S Roura, PhD, ICREC Research Program, Germans Trias i Pujol Health Science Research Institute, Ctra. de Can Ruti, Camí de les Escoles s/n, Badalona 08916, Spain

E-mail: sroura@igtp.cat

${ }^{6}$ These authors contributed equally to this work.

Received 26 June 2016; revised 18 August 2016; accepted 22 August 2016
} 
Quantum Dots (QD), cyanines, and Alexa Fluor dyes, have been reported to partially resist these stringent experimental conditions. ${ }^{4,5}$ However, it is difficult to conjugate QD with antibodies and therefore their use in detecting cell surface antigens is limited. To that end, researchers must usually isolate the cell subset of interest prior to performing flowFISH.

The Brilliant Violet (BV) family of fluorochromes has unique optical properties to produce much brighter fluorescence signal than most of the traditional fluorescent dyes used in flow cytometry. Based on chemistry awarded with Nobel Prize, they are generated by about 1000 fluorescent monomeric subunits per macromolecule that act cooperatively along the entire length of the polymer backbone, resulting in emission of light with high molecular extinction coefficients and high quantum efficiency. ${ }^{6,7}$ Other advantages of this new class of dyes include synthetic modifications of their backbone structure to span the full range of the visible spectrum, and spectral compatibility with each other for multicolor simultaneous labeling. ${ }^{8}$

Heart failure remains a growing medical problem related to major hospitalization, mortality and poor prognosis worldwide. ${ }^{9}$ Although advances in diagnosis and treatment over last decades, many of the pathophysiological mechanisms and prognostic markers remain poorly investigated. In this context, the assessment of monocyte heterogeneity and/or ageing as potentially valuable predicting factors of categories and progression of heart failure is challenging. To that end, standardized instrument settings, reagents, and sample preparation and analytical protocols are required.

Thus, in the present study, we used the newly developed BV fluorochromes to specifically and simultaneously measure the distribution of circulating monocyte subsets and their respective TLs (RTLs) in a cohort of patients with heart failure.

\section{MATERIALS AND METHODS Study Population}

We enrolled 28 consecutive patients diagnosed with heart failure and collected $3 \mathrm{ml}$ of blood into EDTA tubes by conventional forearm venipuncture. The main clinical and demographic characteristics of the patients are summarized in Table 1. The study protocol was approved by the Clinical Research Ethics Committee of our institution, and it conformed to the principles outlined in the Declaration of Helsinki. Written informed consent was previously obtained from each subject. Samples were stored at room temperature and processed within $4 \mathrm{~h}$ after collection.

\section{Multicolor Flow-FISH Procedure} Immunophenotyping

Whole-blood samples were lysed by incubating them with PharmLyse solution (BD Bioscience, San Diego, CA, USA) for $10 \mathrm{~min}$. They were then washed twice with phosphatebuffered saline (PBS; Gibco Life Technologies/Invitrogen,
Table 1 Demographic and clinical characteristics of patients with heart failure

\begin{tabular}{|c|c|}
\hline & $N=28$ \\
\hline Age & $64.9 \pm 12$ \\
\hline Male & $20(71,4 \%)$ \\
\hline \multicolumn{2}{|l|}{ Etiology } \\
\hline Ischemic & $12(42.9 \%)$ \\
\hline IDCM & $6(21.4 \%)$ \\
\hline Hypertensive & $1(3.6 \%)$ \\
\hline Enolic & $1(3.6 \%)$ \\
\hline Toxic & $1(3.6 \%)$ \\
\hline Valvular & $4(14.3 \%)$ \\
\hline Hypertrophic & $2(7.2 \%)$ \\
\hline Others & $1(3.6 \%)$ \\
\hline Duration heart failure (months) & $28(11-72)$ \\
\hline LVEF & $40 \pm 12$ \\
\hline \multicolumn{2}{|l|}{ NYHA classification } \\
\hline$|-| \mid$ & $22(78,6 \%)$ \\
\hline III-IV & $6(21.4 \%)$ \\
\hline Diabetes mellitus & $9(32.1 \%)$ \\
\hline Hypertension & $14(50 \%)$ \\
\hline Previous myocard infarction & $9(32.1 \%)$ \\
\hline \multicolumn{2}{|l|}{ Treatment } \\
\hline ACEI/ARBII & $25(89.3 \%)$ \\
\hline Beta-blockers & $26(92.9 \%)$ \\
\hline MRA & $17(60.7 \%)$ \\
\hline Loop diuretics & $15(53.6 \%)$ \\
\hline Digoxin & $8(28.6 \%)$ \\
\hline Ivabradine & $8(28.6 \%)$ \\
\hline CRT & $3(10.7 \%)$ \\
\hline ICD & $4(14.3 \%)$ \\
\hline
\end{tabular}

Abbrevations: $A C E I$, angiotensin converting enzyme inhibitor; $A R B$, angiotensin II receptor blocker; CRT, cardiac resynchronization therapy; ICD, implantable cardioverter-defibrillator; IDCM, idiopathic dilated cardiomyopathy; LVEF, left ventricular ejection fraction; MRA, mineralocorticoid receptor antagonists; NYHA, New York Heart Association.

Carlsbad, CA, USA) containing $0.5 \%$ bovine serum albumin (Miltenyi Biotech, Bergisch Gladbach, Germany). Cell concentration was determined by flow cytometry using PerfectCount beads (Cytognos, Salamanca, Spain). We stained $1 \times 10^{6}$ cells with titrated amounts of antibodies conjugated to BV or Alexa Fluor dyes and specific to human CD86BV605, CD14-BV785 (Biolegend, San Diego, CA, USA), CD16-BV421 and CD15-AlexaFluor647 (BD Biosciences) in $50 \mu \mathrm{l}$ of Brilliant Stain Buffer (BD Biosciences) for $15 \mathrm{~min}$ at room temperature (RT). We also comparatively tested a battery of commercially available antibodies against human 
Table 2 BD Fortessa SORP optical configuration

\begin{tabular}{lll}
\hline Splitter & BP filter & $\begin{array}{l}\Delta \\
\text { AutoFL }\end{array}$ \\
\hline $\begin{array}{ll}\text { Violet laser }(405 \mathrm{~nm}) \\
750 \text { LP }\end{array}$ & \\
670 LP & $780 / 60$ & Low \\
630 LP & $710 / 50$ & Medium \\
595 LP & $660 / 20$ & High \\
550 LP & $605 / 40$ & High \\
535 LP & $585 / 42$ & High \\
505 LP & $560 / 40$ & High \\
None & $515 / 20$ & High \\
& $450 / 50$ & High
\end{tabular}

$\begin{array}{lll}\text { Blue laser }(488 \mathrm{~nm}) & \\ 750 \mathrm{LP} & 780 / 60 & \text { Medium } \\ 670 \mathrm{LP} & 705 / 70 & \text { Medium } \\ 635 \mathrm{LP} & 670 / 14 & \text { High } \\ 600 \mathrm{LP} & 616 / 23 & \text { High } \\ \text { None } & 575 / 26 & \text { High }\end{array}$

Green laser $(532 \mathrm{~nm})$

$\begin{array}{lll}750 \mathrm{LP} & 780 / 60 & \text { Medium } \\ 670 \mathrm{LP} & 705 / 70 & \text { Medium } \\ 635 \mathrm{LP} & 670 / 14 & \text { High } \\ 600 \mathrm{LP} & 616 / 23 & \text { High } \\ \text { None } & 575 / 26 & \text { High }\end{array}$

\begin{tabular}{lll} 
Red laser $(640 \mathrm{~nm})$ & \\
$735 \mathrm{LP}$ & $780 / 60$ & Low \\
$690 \mathrm{LP}$ & $710 / 50$ & Low \\
None & $660 / 20$ & Low \\
\hline
\end{tabular}

All fluorescence channels are named with the first letter of the laser (B, V, G, and R) and the central wavelength of the band-pass filter. Mirrors-spliters and band-pass filters are shown. Last column lists the level of increase in cellular autofluorescence in FISH protocol: High ( $>5$-fold), Medium (2- to 5fold) and Low $(<2$-fold) regarding background (non-FISH protocol) level.

CD8 coupled to BV421, BV510, BV570, BV605, BV650, BV711, or BV786 (Biolegend; BD Biosciences) or PE, PECy7, APC, PerCP, or BB515 (BD Biosciences) bound to compensation capture beads (OneComp eBeads; eBioscience, San Diego, CA, USA). After a brief centrifugation and two washes in PBS, cells were fixed with $6 \mathrm{mM}$ bis sulfosuccinumidyl suberate (Sigma-Aldrich) for $30 \mathrm{~min}$ at $2-8^{\circ} \mathrm{C}$. The reaction was then immediately quenched by incubating with $1 \mathrm{M}$ Tris buffer ( $\mathrm{pH}$ 8.0) for $15 \mathrm{~min}$ at $\mathrm{RT}$, and residual red blood cells were removed using FACS lysing solution (BD Biosciences) for $7 \mathrm{~min}$ at RT.

\section{In situ hybridization}

FISH was performed with the Telomere PNA kit (Dako, Glostrup, Denmark) in accordance with the manufacturer's instructions and using the lymphoblastic leukemia 1301 cell line as an internal control. An equal volume $(300 \mu \mathrm{l})$ of hybridization solution with or without FITC-labeled PNA probe (telomere-specific peptide nucleic acid probe) was added to sample and control tubes, respectively. Tubes were then hybridized for $10 \mathrm{~min}$ at $82^{\circ} \mathrm{C}$ in a dry block heater (Grant Instruments, Cambridge, UK), and thereafter incubated overnight at RT. Tubes were washed twice in wash solution with previous incubation at $40^{\circ} \mathrm{C}$, and resuspended in $500 \mu \mathrm{l}$ of DNA-staining solution for $2-3 \mathrm{~h}$ at $2-8^{\circ} \mathrm{C}$. Samples were acquired by flow cytometry, and a minimum of 10000 monocytes were collected in each sample tube. Correction for DNA ploidy of the blood sample versus the internal control was performed as described previously. ${ }^{10}$

\section{Flow cytometry analysis}

All samples were acquired on a Fortessa SORP flow cytometer (BD Biosciences) using 20 parameters (two scattering and 18 fluorescence detectors). The cytometer was equipped with four lasers with the following configurations: $100-\mathrm{mW}$ $488 \mathrm{~nm}, 150 \mathrm{~mW} 532 \mathrm{~nm}, 50 \mathrm{~mW} 405 \mathrm{~nm}$, and $100 \mathrm{~mW}$ $640 \mathrm{~nm}$. All fluorescence channels were referred to by the first letter of the laser (B, V, G, and R), and the central wavelength of the band-pass filter (summarized in Table 2). We performed routine daily quality control tests with Cytometer Setup \& Tracking Beads (BD Biosciences) in accordance with the manufacturer's instructions. The initial optimal voltage ranges and linearity for photomultipliers were selected using 6-peak Rainbow Calibration Particles and Unstained CompBeads (BD Biosciences), as described previously. ${ }^{11}$ Owing to the increased cellular autofluorescence associated with FISH, PMT voltages were readjusted to obtain maximum sensitivity and resolution. A compensation matrix was applied by singlefluorochrome controls processed with the flow-FISH protocol. Data were analyzed with FACSDiva (BD Biosciences) and FlowJo (Treestar, Ashland, OR, USA) software.

We initially gated for G0/G1 cells of both leukocyte subsets and 1301 cells based on DNA content and then by scatter properties. Neutrophils and monocytes had similar scatter patterns after FISH; thus, monocytes were sequentially selected based on a CD86 versus CD16 plot, and a CD15 versus CD16 plot to gate out neutrophils. The gated monocytes were then analyzed for CD14 and CD16 expression. Clumped cells were excluded by propidium iodide (PI) area versus PI width plot. Each subset of monocytes and internal control cells was subsequently displayed on a plot comprising the FITC-labeled PNA probe on PI (B695-A) versus the PNA probe (B515-A), and the median fluorescence intensity (MFI) of the PNA probe was calculated. We determined the gate boundaries for CD14- and CD16positive subsets with the appropriate fluorescence minus one controls. For bead-based comparative purposes, beads 
were gated in forward and side scatter plots, and the MFIs of both negative and positive fluorochrome capture beads were analyzed.

The RTL value for each monocyte subset was calculated as the ratio between the MFI of each subset and the MFI of the control cells. Corrections were made for the DNA index of G0/G1 cells, as described previously. ${ }^{12}$

\section{RESULTS}

In these experiments, we assessed whether the newly developed BV fluorochromes were useful for flow-FISH. To that end, we attempted to simultaneously determine the identification of the three mononuclear cell subsets referred to as classical $\left(\mathrm{CD} 14^{+} \mathrm{CD} 16^{-}\right)$, intermediate $\left(\mathrm{CD} 14^{+} \mathrm{CD} 16^{+}\right)$ and nonclassical $\left(\mathrm{CD} 14^{\text {low }} \mathrm{CD} 16^{+}\right)$, as well as their respective RTLs in a cohort of patients with heart failure.

We first tested the thermal stability of BV dyes at $82^{\circ} \mathrm{C}$ and the effects of the extensive fixation step that takes place in standard FISH protocols. We did so by analyzing the fluorescence emissions of different commercially available BV fluorochromes coupled to antibody capture beads. This allowed us not only to exclude the increased cellular autofluorescence that occurs during FISH but also to avoid changes in the antigen-antibody complex that can be provoked by the harsh conditions associated with FISH. The polymer-based BV dyes were heat stable and withstood the stringent conditions of hybridization with no degradation or loss of emission (Figures 1a-g). Additionally, the recently developed polymer-derived fluorochrome Brilliant Blue 515 showed the same thermal resistance (Figure 1h). By contrast, conventional organic or protein-based fluorochromes such as phycoerythrin, allophycocyanin, and peridinin-chlorophyll protein and related tandem fluorochromes were commonly completely degraded and their emissions abrogated during FISH (Figures 1I and k).

To determine if the stability of the BVs was sustained in a cell-based protocol, we incubated whole-blood leukocytes extracted from patients with titrated amounts of anti-human CD8-BV421, CD14-BV786, CD15-Alexa647, CD16-BV421, and CD86-BV605, and assessed them by standard FISH procedures. Although several of the photomultipliers that we evaluated in the violet laser line showed an increase from medium to high levels of cellular autofluorescence after fixation and incubation at $82{ }^{\circ} \mathrm{C}$ (most pronounced between wavelengths 515 and $660 \mathrm{~nm}$; Table 2), discrimination among $\mathrm{CD}^{+}, \mathrm{CD} 4^{+}, \mathrm{CD} 5^{+}, \mathrm{CD}_{1}{ }^{+}$, and $\mathrm{CD} 86^{+}$cell subsets related to the nonhybridization protocol was preserved (Figure 2). Table 2 shows the increased cellular autofluorescence associated with the FISH protocol. Increases in cellular autofluorescence were classified as High ( $>5$-fold), Medium (2- to 5 -fold), or Low ( $<2$-fold) with respect to background levels. All of the BV dyes retained $>60 \%$ of their pre-FISH Stain Index (data not shown).

We next assessed whether using BV-conjugated antibodies would allow us to discriminate among the different monocyte subsets circulating in patients' blood by measuring cell surface expression of CD14 and CD16. We found that the BVconjugated antibodies successfully discriminated among the monocyte subsets (Figure 3). Moreover, percentages of monocyte subsets measured before (after stopping BS3 crosslinking with Tris buffer) and after hybridization were very similar when measured with CD15-AlexaFluor647, CD16-BV421, CD86-BV605, and CD14-BV785, all of which are spectrally compatible with FITC-labeled PNA and PI (Figure 3). The calculated RLTs corresponding to each monocyte subset were $9.68 \pm 2.21,9.76 \pm 2.26$, and $9.96 \pm 2.20$ for classical, intermediate, and nonclassical monocytes, respectively (Figure 4).

\section{DISCUSSION}

In the present study, we evaluated whether BVs, a novel family of polymer-based fluorescent dyes, are valuable as heat-stable alternatives to conventional organic or proteinbased fluorochromes in flow-FISH. We used the BVs to simultaneously measure the different circulating monocyte subpopulations and their respective TLs in blood samples from a cohort of patients with heart failure.

In general, current flow-FISH measurement of TL requires targeted cell purification (eg, by cell sorting). ${ }^{13}$ As an alternative, some studies have attempted to identify cell subsets by immunophenotyping and then applying TL calculations to all cells in a heterogeneous sample such as venous blood. ${ }^{14}$ Other studies have used a variety of heatstable fluorochromes. ${ }^{15}$ In protocols in which targeted cells must be isolated prior to flow-FISH measurement of TL, it is also difficult to obtain the necessary number of cells for TL. Other limitations include thermal instability of conventional fluorochromes and high levels of cellular autofluorescence during the analytical process. ${ }^{16}$ Although other fluorochromes such as QD, cyanines, and Alexa Fluor are relatively thermally stable under the harsh conditions required for hybridization, their implementation is limited by the scarcity of appropriate reagents, the difficulty associated with conjugating them to antibodies, their excitation by several wavelengths of lasers, ${ }^{17}$ and the overlap of cellular autofluorescence and dye emissions.

Their polymeric structure and their benefit in multicolor staining panels suggest that the BV family of dyes may be successful alternatives to the brightest fluorochromes commonly used for immunophenotyping purposes, such as phycoerythrin and allophycocyanin. Moreover, their use enables the violet laser for polychromatic excitations. Additional major advantages of BV-based flow cytometry include their high solubility; lack of nonspecific binding; compatibility with intracellular staining; and efficient, stable conjugation to antibodies. ${ }^{6} \mathrm{BV}$ dyes are more thermally stable than most low molecular weight fluorochromes, which lose their fluorescence under the intense fixation and high temperature $\left(82^{\circ} \mathrm{C}\right)$ required for DNA denaturation and hybridization of the fluorescent telomere probe. 
a

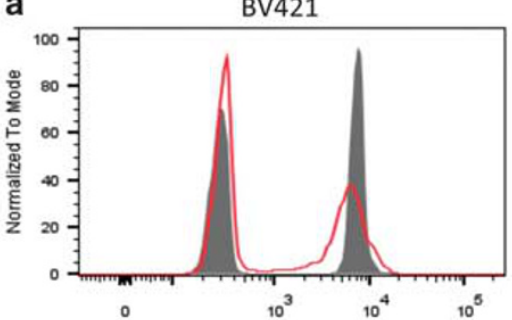

b

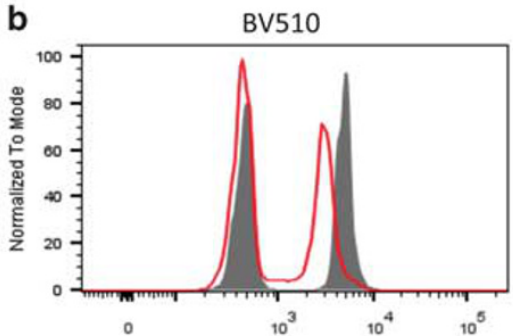

C

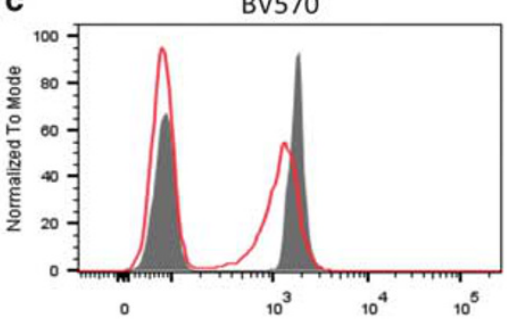

d

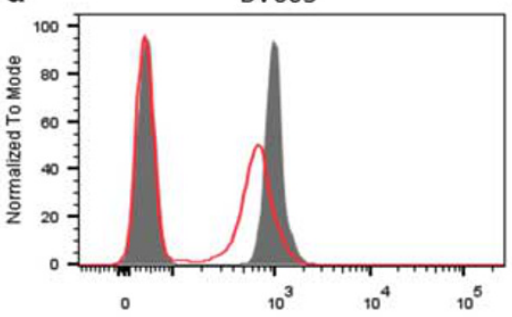

e

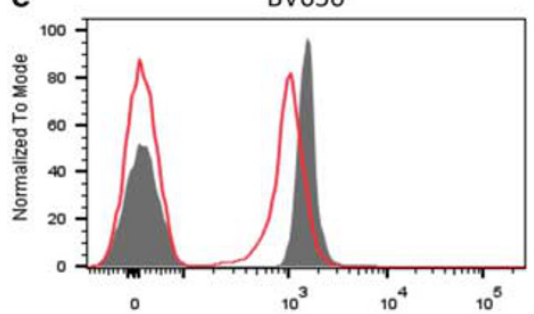

f

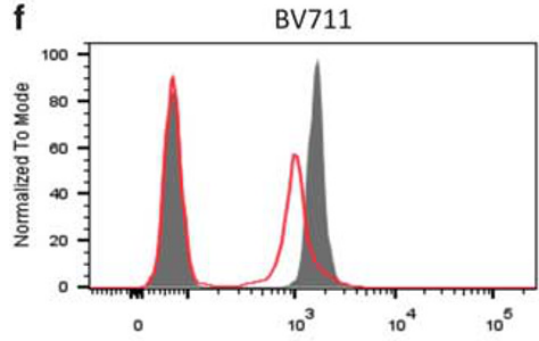

g

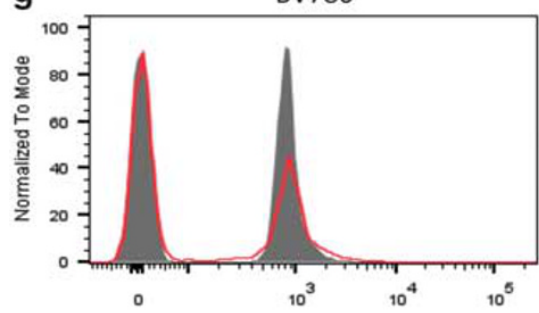

h

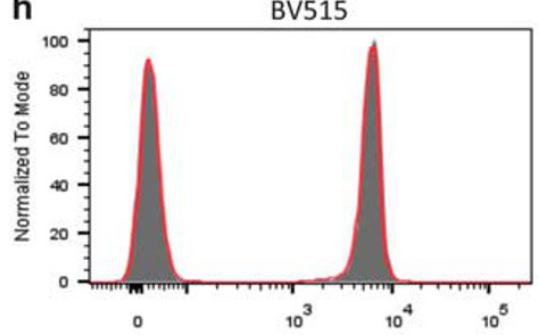

i

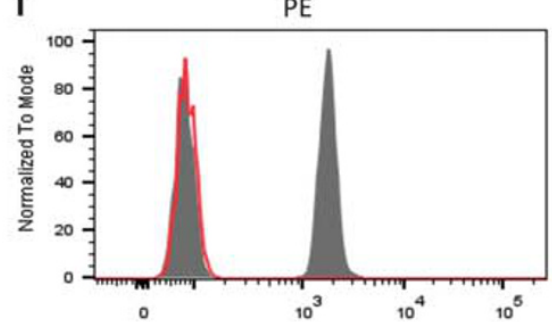

j

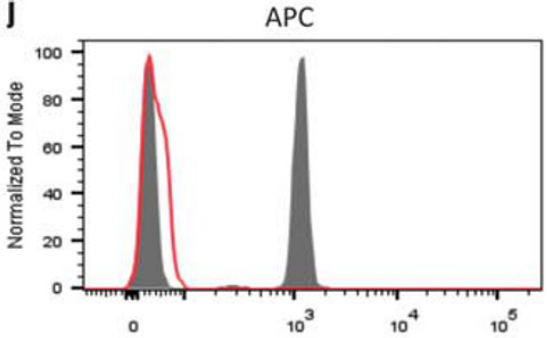

k

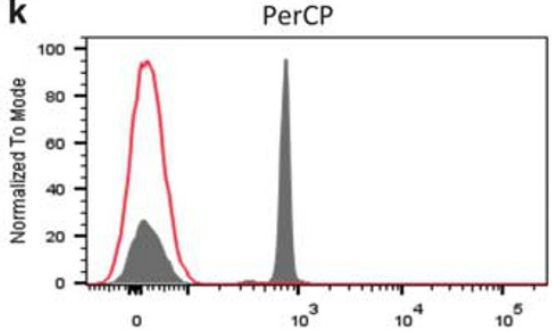

Figure 1 Direct effect of FISH protocol on Brilliant Violet fluorochromes. Detection of BV421 (a), BV510 (b), BV570 (c), BV605 (d), BV650 (e), BV711 (f), BV786 (g) and BB515 (h) fluorescence signal using dye-labeled (right peak) and unlabeled (left peak) beads. Each BV signal is compared in FISH (red line histograms) and in non-FISH conditions (gray filled histograms). In contrast, conventional organic fluorochromes such as phycoerythrin (i), allophycocyanin (j) and peridinin chlorophyll (k) suffer complete degradation and subsequent emission abrogation under FISH conditions.

Based on the qualities described above, we hypothesized that BVs, as well as the new polymer-based dye Brilliant Blue BB515, would be appropriate alternatives to conventional fluorochromes in assays designed to simultaneously identify specific cell subpopulations (immunophenotyping) and hybridize probes to telomeres in situ. We also tested stabilization of the fluorochrome-antibody complex with the amine-to-amine cross-linker bis sulfosuccinumidyl suberate. This cross-linker is homobifunctional, water-soluble, noncleavable, and membrane impermeable; all of these properties are essential for BV-derived prehybridization fluorescence emission. ${ }^{18}$

To evaluate which BV dyes perform best in the detection of CD14, CD16, and CD86 expression in monocyte subsets and
TL calculation, we measured the levels of cellular autofluorescence induced by treatment and the spectral overlap of DNA dyes required for the identification of cell cycle phases. In our hands, PI staining decreases sensitivity in V560-V660 channels (data not shown), and this was taken into account. Moreover, fluorochrome selection must be optimal relative to expected antigen densities and dye efficiencies. ${ }^{19}$ Thus, in the present study, CD16-BV421, CD86-BV605, and CD14-BV786 were chosen for scrutiny.

Monocyte subsets play an important role in inflammation and innate and adapted immunity, and their contribution to cardiovascular diseases is currently being researched. ${ }^{20-23}$ In this context, our aim was to identify alternative fluorochromes for use in multicolor flow-FISH to differentiate 
a

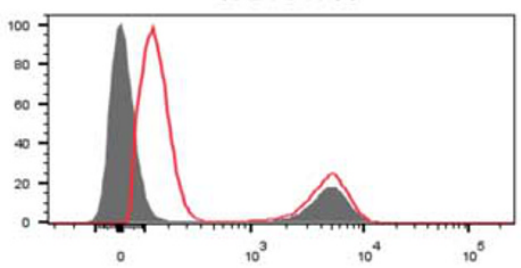

b

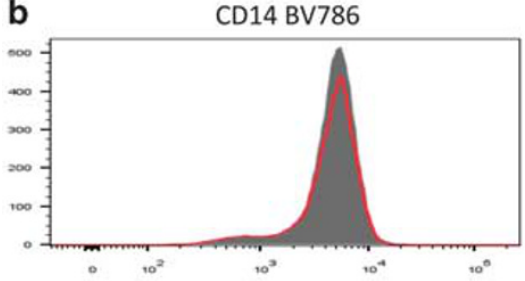

e

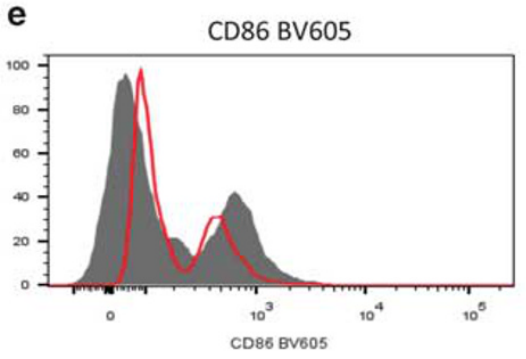

C

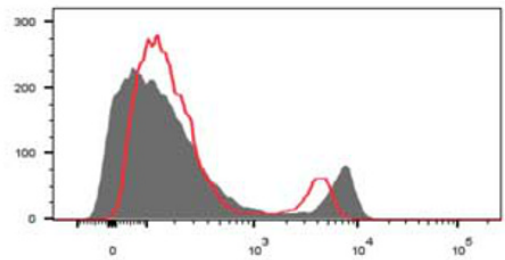

d

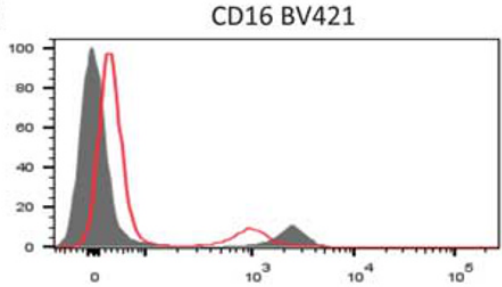

f

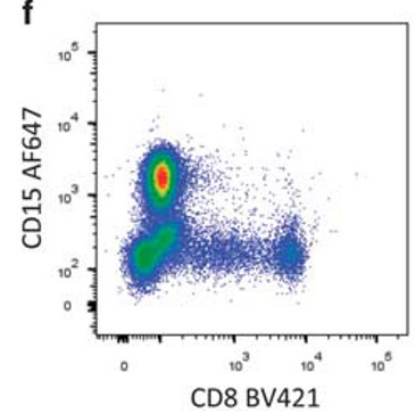

Figure 2 Comparative nonhybridization (gray filled histograms) and hybridization (red line histograms) staining protocols. Peripheral blood mononuclear cells (a) and monocytes (b) stained with CD14-BV786. Although increased cellular autofluorescence is shown using the FISH protocol (a), CD14 ${ }^{+}$cell staining is unaffected (a, b). Monocytes (c) and lymphocytes (d) stained with CD16-BV421. Both cell populations can be clearly discriminated from background. Peripheral blood mononuclear cells stained with CD86-BV605 (e). FISH protocol in a whole-blood sample using heat-stable BV421 and Alexa647-conjugated antibodies for specific detection of $\mathrm{CD} 8^{+}$lymphocytes and $\mathrm{CD} 15^{+}$neutrophils (f).

PRE-HYBRIDIZATION

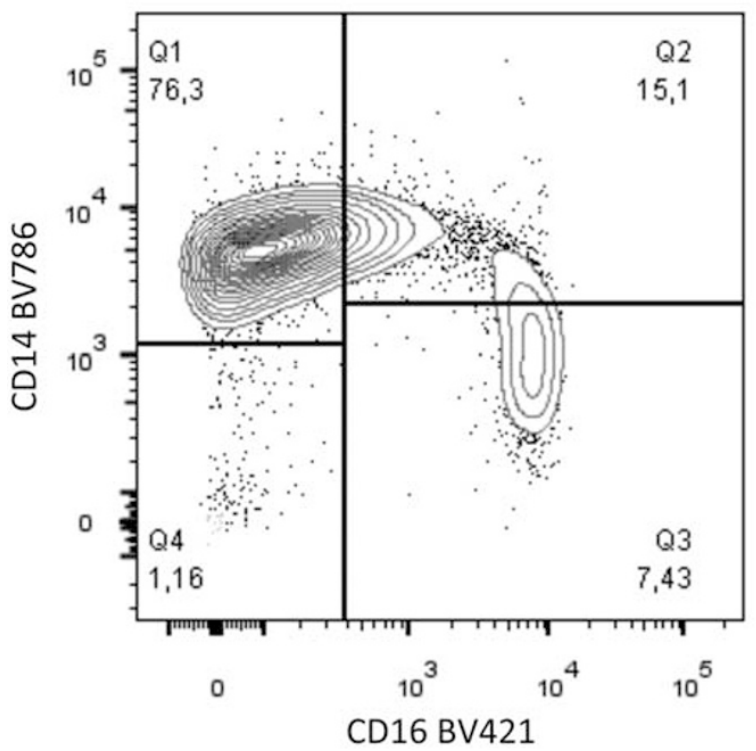

\section{POST-HYBRIDIZATION}

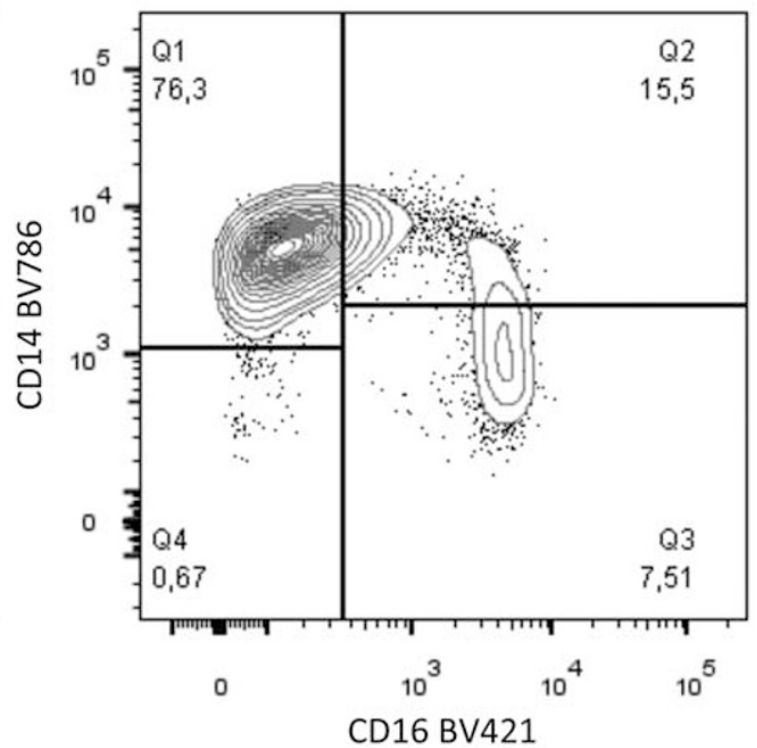

Figure 3 Representative analysis of whole-blood sample from a patient with heart failure. Monocyte subsets showing different expression levels of CD14 and CD16 cell surface antigens under prehybridization (left contour plot) and post-hybridization (right contour plot) conditions. Both plots depict a similar fluorescence intensity and frequency pattern of the different monocyte subsets. 

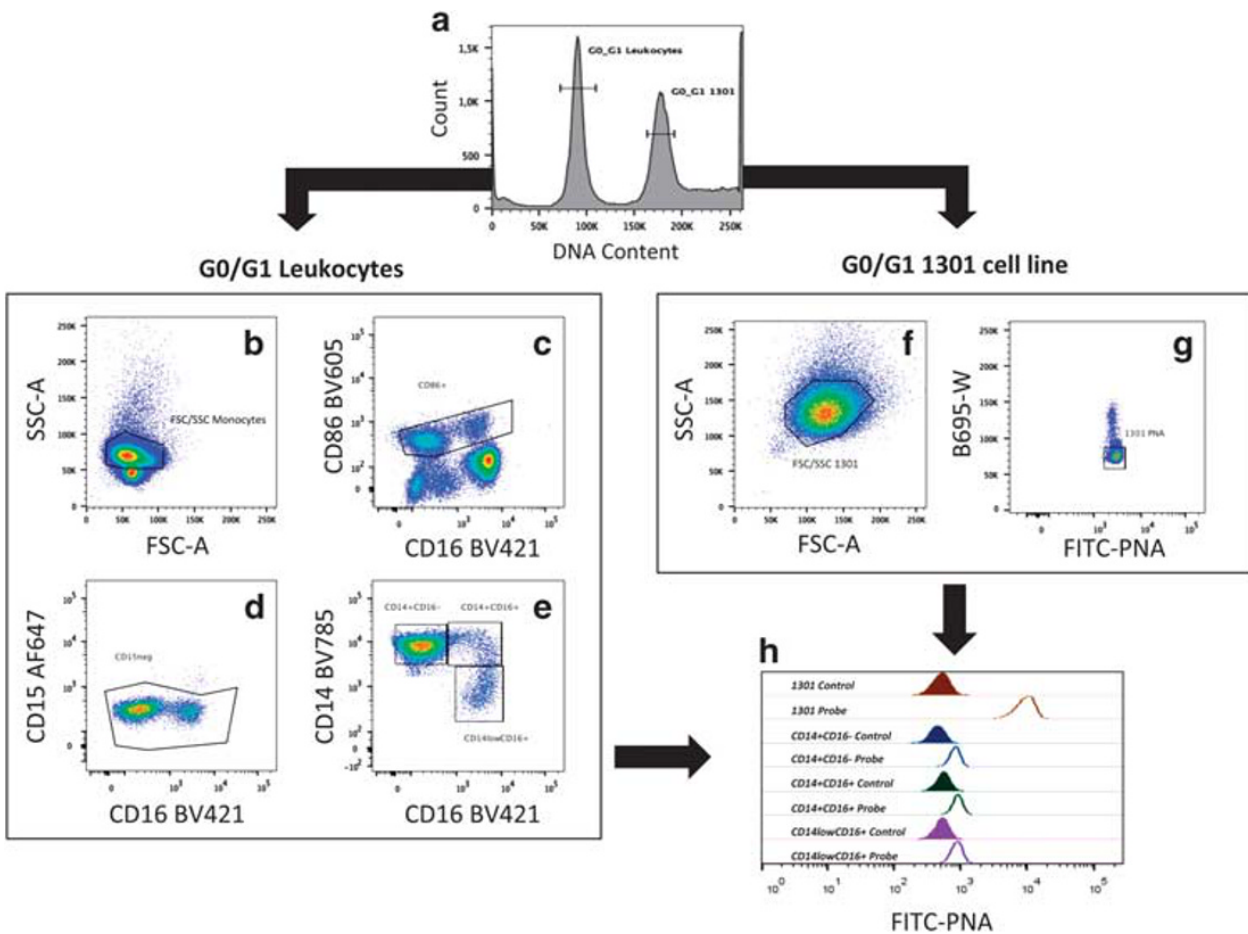

Figure 4 Gating strategy for the RTL analysis in monocyte subsets. G0/G1 cells were selected in DNA content histogram (a) and then gated by scatter properties (b, monocytes and f, 1301 cell line). CD86 versus CD16 (c) and CD15 versus CD16 (d) plots were used to specifically select monocytes; thereafter both CD14 and CD16 expression were analyzed (e). (h) Hybridization of FITC-labeled PNA probe and control (without probe) in monocyte subsets based on the CD4 and CD16 expression and in 1301 cell line, which was used as internal reference control (g).

among monocyte subpopulations and quantify their RTLs. Moreover, fixation steps can lead to loss of differentiation based on scatter properties, and monocyte subsets expressing CD14 and CD16 antigens are not easily separated from other $\mathrm{CD} 6^{+}$leukocytes such as neutrophils. To address this concern, we confirmed that discrimination between monocytes and neutrophils requires the employment of additional cell surface antigens, such as an exclusion marker for neutrophils (eg, CD15).

Taken together, our results show that BVs used in flowFISH can facilitate accurate, specific, relatively inexpensive (approximately 3-fold less expensive), and faster measurement of circulating monocyte subsets and their RTLs in the context of heart failure. Therefore, our technical protocol may be used to further explore important associations among patient categories, disease progression, monocyte heterogeneity, and aging in series that comprise broader ranges of patients than those assessed in conventional studies.

\section{ACKNOWLEDGMENTS}

We specially thank Beatriz González, Margarita Rodríguez, Carmen Rivas, Nuria Benito and Alba Ros for data collection and invaluable work in the Heart Failure Clinic, and Carolina Gálvez Montón for artwork graphs and figures. We also express gratitude to IGTP Biobank for blood samples management. This work was supported by grants from the Ministerio de Educación y Ciencia (SAF2014-59892), Fundació La MARATÓ de TV3 (122232, 201502-30, 20151610), Fundació Daniel Bravo Andreu, Sociedad Española de Cardiología,
Societat Catalana de Cardiologia, Generalitat de Catalunya (SGR 2014), and Acadèmia de Ciències Mèdiques i de la Salut de Catalunya i de Balears. The study was also funded by the Red de Terapia Celular - TerCel (RD12/0019/ 0029), Red de Investigación Cardiovascular - RIC (RD12/0042/0047), and Fondo de Investigación Sanitaria, Instituto de Salud Carlos III (FIS PI14/01682) projects as part of the Plan Nacional de I+D+I and cofounded by ISCIIISubdirección General de Evaluación y el Fondo Europeo de Desarrollo Regional (FEDER).

\section{DISCLOSURE/CONFLICT OF INTEREST}

The authors declare no conflict of interest.

1. Aubert G, Hills $\mathrm{H}$, Lansdorp PM. Telomere length measurement-caveats and a critical assessment of the available technologies and tools. Mutat Res 2012;730:59-67.

2. Rufer N, Dragowska W, Thornbury G, et al. Telomere length dynamics in human lymphocyte subpopulations measured by flow cytometry. Nat Biotechnol 1998;16:743-747.

3. Gutierrez-Rodrigues F, Santana-Lemos BA, Scheucher PS, et al. Direct comparison of flow-FISH and GPCR as diagnostic tests for telomere length measurement in humans. PLoS One 2014;9:11.

4. Schmid I, Dagarag MD, Hausner MA, et al. Simultaneous flow cytometric analysis of two cell surface markers, telomere length, and DNA content. Cytometry 2002;49:96-105.

5. Kapoor V, Hakim FT, Rehman N, et al. Quantum dots thermal stability improves simultaneous phenotype-specific telomere length measurement by FISH-flow cytometry. J Immunol Methods 2009;344:6-14.

6. Hide F, Diaz-Garcia M, Schwartz B, et al. New developments in the photonic applications of conjugated polymers. Acc Chem Res 1997;30: 430-436.

7. Heeger PS, Heeger AJ. Making sense of polymer-based biosensors. Proc Natl Acad Sci USA 1999;96:12219-12221. 
8. Chattopadhyay PK, Gaylord B, Palmer A, et al. Brilliant violet fluorophores: a new class of ultrabright fluorescent compounds for immunofluorescence experiments. Cytometry A 2012;81:456-466.

9. Ponikowski P, Voors AA, Anker SD et al. Authors/Task Force Members; Document Reviewers. 2016 ESC Guidelines for the diagnosis and treatment of acute and chronic heart failure: The Task Force for the diagnosis and treatment of acute and chronic heart failure of the European Society of Cardiology (ESC). Developed with the special contribution of the Heart Failure Association (HFA) of the ESC. Eur J Heart Fail 2016;18:891-975.

10. Vindeløv LL, Christensen IJ, Jensen G, et al. Limits of detection of nuclear DNA abnormalities by flow cytometric DNA analysis. Results obtained by a set of methods for sample-storage, staining and internal standardization. Cytometry 1983;3:332-339.

11. Perfetto SP, Ambrozak D, Nguyen R, et al. Quality assurance for polychromatic flow cytometry. Nat Protoc 2006;1:1522-1530.

12. Hultdin $M$, Grönlund $E$, Norrback KF, et al. Telomere analysis by fluorescence in situ hybridization and flow cytometry. Nucleic Acids Res 1998;26:3651-3656.

13. Armengol MP, Sabater L, Fernández M, et al. Influx of recent thymic emigrants into autoimmune thyroid disease glands in humans. Clin Exp Immunol 2008;153:338-350.

14. Haver VG, Hartman MH, Mateo Leach I, et al. Leukocyte telomere length and left ventricular function after acute ST-elevation myocardial infarction: data from the glycometabolic intervention as adjunct to primary coronary intervention in ST elevation myocardial infarction (GIPS-III) trial. Clin Res Cardiol 2015;104:812-821.

15. Spyridopoulos I, Hoffmann J, Aicher A, et al. Accelerated telomere shortening in leukocyte subpopulations of patients with coronary heart disease: role of cytomegalovirus seropositivity. Circulation 2009;120:1364-1372.

16. Mosiman VL, Patterson BK, Canterero $\mathrm{L}$, et al. Reducing cellular autofluorescence in flow cytometry: an in situ method. Cytometry 1997;30:151-156.

17. Chattopadhyay PK, Perfetto SP, Yu J, et al. The use of quantum dot nanocrystals in multicolor flow cytometry. Wiley Interdiscip Rev Nanomed Nanobiotechnol 2010;2:334-348.

18. Batliwalla FM, Damle RN, Metz C, et al. Simultaneous flow cytometric analysis of cell surface markers and telomere length: analysis of human tonsilar B cells. J Immunol Methods 2001;247:103-109.

19. Mahnke YD, Roederer M. Optimizing a multicolor immunophenotyping assay. Clin Lab Med 2007;27:469-485.

20. Heimbeck I, Hofer TP, Eder C, et al. Standardized single-platform assay for human monocyte subpopulations: lower CD14+CD16++ monocytes in females. Cytometry A 2010;77:823-830.

21. Libby $P$, Nahrendorf $M$, Swirski FK. Monocyte heterogeneity in cardiovascular disease. Semin Immunopathol 2013;35:553-562.

22. Nahrendorf M, Swirski FK. Monocyte and macrophage heterogeneity in the heart. Circ Res 2013;112:1624-1633.

23. Woollard KJ, Geissmann F. Monocytes in atherosclerosis: subsets and functions. Nat Rev Cardiol 2010;7:77-86. 